\title{
Performance of activated carbons in consecutive phenol photooxidation cycles
}

Leticia F. Velasco ${ }^{1,2}$, Rocio J. Carmona ${ }^{1}$, Juan Matos $^{3}$, Conchi O. Ania ${ }^{1 *}$

${ }^{1}$ Adsorption and Environmental Remediation on Porous Solids (ADPOR), Dpt. Chemical Processes for Energy and Environment, Instituto Nacional del Carbón, INCAR-CSIC, Apdo. 73, 33080 Oviedo, Spain

${ }^{2}$ Dpt. Chemistry, Royal Military Academy, Renaissancelaan 30, 1000 Brussels, Belgium

${ }^{3}$ Dpt. Photocatalysis and Alternative Energies, Venezuelan Institute for Scientific Research (IVIC), 20632, Caracas 1020-A, Venezuela

\begin{abstract}
The long term performance of semiconductor-free activated carbons showing photochemical activity was explored by monitoring the photodegradation of phenol from aqueous solution along 20 hours of illumination in consecutive photocatalytic cycles. The efficiency of the process was evaluated in terms of phenol conversion, mineralization degree and evaluation of degradation intermediates upon cycling. Data showed a strong dependence of the photooxidation efficiency on the hydrophobic/hydrophilic nature of the carbons. The outstanding role of dissolved oxygen as a promoter of phenol photodegradation through the formation of O-radicals upon illumination of the carbons was also demonstrated. The excess of oxygen not only improved phenol conversion and mineralization, but delayed the clogging of the carbon's porosity upon cycling. This is important since a fraction of the photooxidation reaction also takes place inside the porous network of the carbon materials. Overall, the performance of the activated carbons, especially in conditions of excess of oxygen, is comparable to that of commercial titania.
\end{abstract}

*Corresponding author. Tel./Fax: +34 985 118846/+34 985 297662. E-mail address: conchi.ania@incar.csic.es (C.O. Ania) 


\section{Introduction}

Photochemical reactions are useful for the degradation of refractory pollutants, since the excitation of electronic molecular states at energies provided by light may induce chemical bond breaking [1]. However, the large-scale implementation of photocatalytic processes in environmental remediation is yet hindered by technological and economical drawbacks (mainly low semiconductor activity under visible light, high recombination rate of photogenerated electron-hole pairs, and recovery and reutilization issues) [2]. Addressing these problems calls out for a research to be conducted to enhance the performance of semiconductors or to explore the possible use of other types of materials in this kind of applications. Among different approaches, the use of carbon materials in heterogeneous photocatalysis has attracted a lot of research efforts during the last years [3-8]. First investigations in the field focused on the use of carbons as supports or additives of $\mathrm{TiO}_{2}$, and the enhanced photocatalytic performance of carbon $/ \mathrm{TiO}_{2}$ composites has been attributed to several factors associated to visible light absorption and the porosity the carbon support, and/or strong interfacial electronic effects [3-8].

More recently, our previous studies have demonstrated the self-photochemical activity of certain activated carbons under irradiation in the absence of conventional semiconductors and their ability to generate O-radical species upon UV irradiation [9-12]. Despite the increasing interest in the topic, there is still a dearth in the understanding of the origin of the carbon/light interactions accounting for the formation of the O-radicals and their enhanced performance under UV light. Although further studies are needed to establish the origin of the photochemical behavior of activated carbons, it is also necessary to corroborate their activity trough the performance of consecutive photodegradation runs. In order to be competitive with other materials, activated carbons for photo-oxidation must fulfill the requirements of long cycle life, and good degradation efficiency. Thus, the objective of this work was to explore the potential application of activated carbons showing photochemical activity in wastewater remediation [9], by investigating their performance towards the photocatalytic degradation of phenol from solution after several consecutives runs, and their stability under prolonged exposure to UV light (ca. $20 \mathrm{~h}$ ). Scarce studies are available in the literature dealing with the long-term cycling performance of photocatalysts, including our previous data on carbon/titania composites [13]. Moreover, to the best of our knowledge this is the first time that the response of semiconductor-free activated carbons as photocatalysts are tested through the performance of consecutive photocatalytic runs. 
The choice of the activated carbons was based on our previous works reporting their good performance towards phenol photooxidation in the absence of semiconductors after 6 hours of irradiation [9]. Moreover, their rich surface chemistry allowed to study the influence of the carbon functionalization on their long-term stability and photodegradation performance under UV light, compared to that of commercial $\mathrm{TiO}_{2}$ under similar experimental conditions. The efficiency of the systems was evaluated in terms of phenol conversion and mineralization degree upon consecutive cycles; the evolution of the main phenol photodegradation intermediates was also assessed upon cycling. Additionally, the role of the oxygen as a promoter of phenol photooxidation was studied. Experiments have been carried out for 20 hours of illumination, and data showed that the performance of the activated carbons, especially in conditions of excess of oxygen, is still comparable to that of commercial titania. Although the results obtained can be very dependent on the nature of the carbons investigated and further studies would be needed using other carbon materials, we expect that the general trend would be the same for other carbons showing similar characteristics (concerning surface functionalization, hydrophobicity, pore texture and phenol photodegradation yields).

\section{Experimental}

\subsection{Materials}

A commercial powdered lignocellulose-based activated carbon (sample CV, phosphoric acid activation) and with a mean particle size below $45 \mu \mathrm{m}$ was selected for this study [9]. A second sample (labeled as $\mathrm{CVH}$ ) was prepared by thermal treatment at $850^{\circ} \mathrm{C}$ under inert atmosphere (30 $\mathrm{min}, \mathrm{N}_{2}$ flow $50 \mathrm{ml} / \mathrm{min}$ ) of carbon $\mathrm{CV}$ to remove the surface functionalities, thus obtaining a more hydrophobic material. For the sake of comparison, commercially available titania powders (Evonik, P25) were also used as a reference photocatalyst in this kind of applications. The main physicochemical characteristics of the tested materials are compiled in Table 1 and Fig. S1 and S2 in Suppl. Info.

\subsection{Photodegradation runs}

Photodegradation experiments were carried out at room temperature using a photo-reactor of $400 \mathrm{~mL}$ capacity and a loading catalyst ratio of $0.5 \mathrm{~g} / \mathrm{L}$. The UV irradiation source was provided by a high pressure mercury lamp (Helios Italquartz, $125 \mathrm{~W}$, emitting at 313, 360, 404, 436, 546, 577 and $579 \mathrm{~nm}$ ), vertically suspended in a cylindrical, double-walled quartz jacket cooled by flowing water, immersed in the solution. The water cell was used to control the temperature during the experiments, preventing any overheating of the suspension due to 
the irradiation. In each run, about $200 \mathrm{mg}$ of the catalysts (either carbon or titania) were added to $400 \mathrm{~mL}$ of phenol solution under continuous and vigorous stirring (900 rpm); then the suspensions were allowed to equilibrate under dark conditions before being illuminated. After the equilibration step, the suspension was irradiated for $180 \mathrm{~min}$.

Table 1. Main physicochemical characteristics of the selected activated carbons obtained from gas adsorption, elemental analysis, point of zero charge, Boehm titration and TPD-MS.

\begin{tabular}{|c|c|c|c|}
\hline & $\overline{\mathbf{C V}}$ & $\mathrm{CVH}$ & $\mathrm{TiO}_{2}$ \\
\hline $\mathrm{S}_{\mathrm{BET}}\left[\mathrm{m}^{2} \mathrm{~g}^{-1}\right]$ & 1280 & 1040 & 53 \\
\hline$V_{\text {TOTAL }}\left[\mathrm{cm}^{3} \mathbf{g}^{-1}\right]^{\mathrm{a}}$ & 1.014 & 0.787 & 0.083 \\
\hline$V_{\text {MICROPORES }}\left[\mathrm{cm}^{3} \mathrm{~g}^{-1}\right]^{\mathbf{b}}$ & 0.314 & 0.272 & -- \\
\hline$V_{\text {MESOPORES }}\left[\mathrm{cm}^{3} \mathrm{~g}^{-1}\right]^{b}$ & 0.517 & 0.357 & -- \\
\hline C [wt.\% $]^{c}$ & 84.6 & 90.9 & -- \\
\hline$H[w t . \%]^{c}$ & 2.4 & 0.9 & -- \\
\hline $\mathrm{N}$ [wt.\% $]^{\mathrm{c}}$ & 0.1 & 0.1 & -- \\
\hline S [wt.\%] & -- & -- & -- \\
\hline $\mathrm{O}[\mathrm{wt.} \%]^{\mathrm{c}}$ & 12.0 & 5.9 & -- \\
\hline Ash [wt.\% $]^{c}$ & 0.9 & 2.2 & -- \\
\hline pH $H_{P Z C}$ & 2.2 & 6.5 & 5.2 \\
\hline Amount $\mathrm{CO}_{2}[\mathrm{mmol} / \mathrm{g}]^{\mathrm{d}}$ & 0.45 & 1.93 & -- \\
\hline Amount CO [mmol/g] ${ }^{\mathrm{d}}$ & 0.06 & 0.51 & - \\
\hline Carboxilic groups [meq/g] ${ }^{\mathrm{e}}$ & 0.41 & 0.07 & -- \\
\hline Lactones $[\mathrm{meq} / \mathrm{g}]{ }^{\mathrm{e}}$ & 0.31 & 0.12 & -- \\
\hline Phenolic groups [meq/g] ${ }^{\text {e }}$ & n.d. & 0.04 & -- \\
\hline Total Acidic groups [meq/g] ${ }^{\mathrm{e}}$ & 0.72 & 0.23 & -- \\
\hline Total Basic groups [meq/g] ${ }^{\mathrm{e}}$ & n.d. & 0.24 & -- \\
\hline \multicolumn{4}{|c|}{$\overline{\mathrm{a}}$ evaluated at relative pressure $\sim 0.95$} \\
\hline \multicolumn{4}{|c|}{ b evaluated from DFT method applied to $N_{2}$ adsorption data } \\
\hline \multicolumn{4}{|l|}{ d quantification from TPD-MS } \\
\hline${ }^{\mathbf{e}}$ Boehm titration (n.d., not detec & & & \\
\hline
\end{tabular}

Since we are using porous carbons, to maintain the same phenol concentration in solution at the beginning of each cycle (ca. $50 \mathrm{ppm}$ ), a pre-equilibration step at dark conditions was 
carried out (Fig. 1). Phenol adsorption kinetics and capacity of the activated carbons were previously evaluated at dark conditions to establish the time required for the equilibrium (ca. $30 \mathrm{~min}$ in all the samples) and the amount adsorbed. Hence before the irradiation was applied, $\mathrm{CV}$ and $\mathrm{CVH}$ carbons were allowed to pre-equilibrate with phenol solutions of 65 and $78 \mathrm{ppm}$ concentration, respectively. Furthermore, to maintain the same initial concentration and volume of phenol solution at the beginning of each cycle, $5 \mathrm{~mL}$ of a concentrated phenol solution (ca. 1500-2500 ppm, depending on the carbon) were added to the photoreactor in order to begin the next photocatalytic run in similar conditions than the previous one.

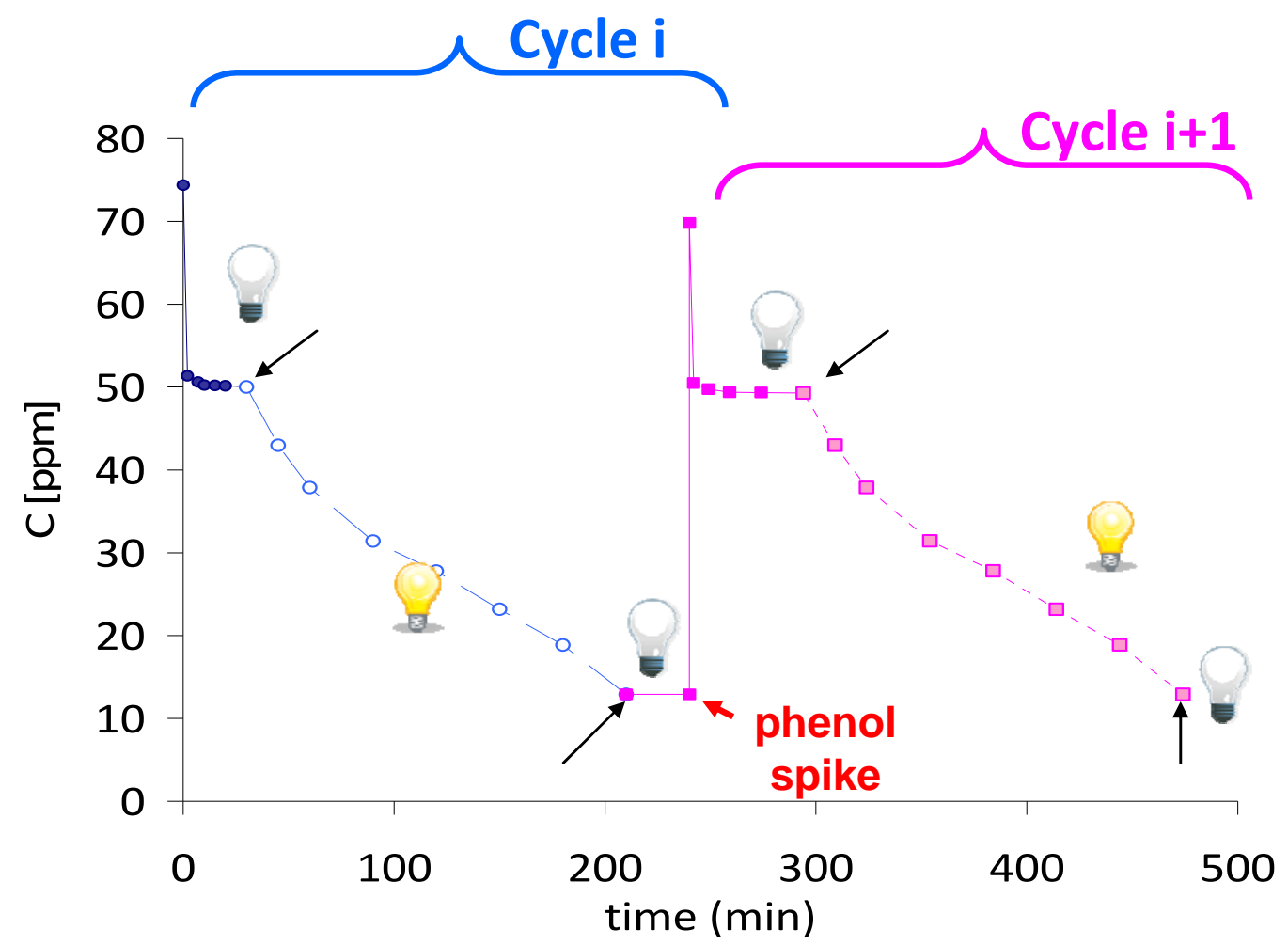

Fig. 1 . Sketch illustrating the experimental procedure followed for the consecutive phenol photooxidation cycles on sample CVH. Arrows indicate the beginning/end of the irradiation period.

Small aliquots of the solution $(\sim 1 \mathrm{~mL})$ were taken out at predetermined time intervals and analyzed by reverse-phase HPLC (Spherisorb C18 column $125 \mathrm{~mm}$ x $4 \mathrm{~mm}$, methanol to water $5: 95,30^{\circ} \mathrm{C}, 0.7 \mathrm{ml} / \mathrm{min}$ flow rate, photodiode array detector). The samples were previously filtered using regenerated cellulose filter having mean pore size of $0.45 \mu \mathrm{m}$. Total organic carbon (TOC) of the solution at the end of each run was also measured in a TOC-V analyzer. To keep constant the initial concentration for each photocatalytic cycle, a spike of a concentrated phenol solution (recalculated from the amount of phenol remaining in solution) 
was added to the suspension; subsequently, the next run was carried out under similar conditions of equilibration for $30 \mathrm{~min}$ under dark followed by illumination for $180 \mathrm{~min}$. Photodegradation cycles were carried out in the presence of air, under ambient conditions (no external air supply) and $\mathrm{O}_{2}$ saturation conditions (provided by continuous air bubbling through the solution). Direct photolysis (non-catalyzed reaction) of phenol was performed through the irradiation of an aqueous solution of phenol in the absence of catalyst. All the experiments were done in triplicate with deviations below $5 \%$ in all cases; reported data represent the average values.

\subsection{Textural and Chemical Characterization}

The porous texture of the samples was characterized by measuring the $\mathrm{N}_{2}$ adsorption isotherms at $-196{ }^{\circ} \mathrm{C}$ (ASAP 2010, Micromeritics) before and after the consecutive photocatalytic cycles. Before the experiments, the samples were outgassed under vacuum (ca. $10^{-3}$ torr) at $120^{\circ} \mathrm{C}$ overnight. The isotherms were used to calculate the specific surface area, $\mathrm{S}_{\mathrm{BET}}$, total pore volume, $\mathrm{V}_{\mathrm{T}}$, and pore volumes using the $\mathrm{DR}$ formulism and non-local density functional theory (DFT). Elemental analysis was carried out in LECO CHNS-932 and LECO VTF-900 automatic analyzers. The surface chemistry of the carbons was further characterized by temperature programmed desorption (TPD) analysis and Boehm titration. TPD experiments were performed with samples of about $100 \mathrm{mg}$ in a Chemisorb 2750 apparatus (Micromeritics), under an argon flow rate of $50 \mathrm{~cm}^{3} \mathrm{~min}^{-1}$, at a heating rate of $15{ }^{\circ} \mathrm{C} \mathrm{min}{ }^{-1}$, up to a final temperature of $900{ }^{\circ} \mathrm{C}$. The amounts of $\mathrm{CO}$ and $\mathrm{CO}_{2}$ evolved during the experiment were quantified by means of a mass quadrupole (Balterzs). Boehm method was carried out by neutralization with bases of increasing strength: $\mathrm{NaHCO}_{3}, \mathrm{Na}_{2} \mathrm{CO}_{3}$ and $\mathrm{NaOH}$ [14]. About $0.5 \mathrm{~g}$ of the carbon was put in contact with $100 \mathrm{ml}$ of $0.05 \mathrm{~N}$ base solution in sealed flasks. The suspensions were shaken for $16 \mathrm{~h}$ and then filtered. The excess of base remaining in the solution was determined from back titration after adding an excess of standard $\mathrm{HCl}$ solution. It was assumed that $\mathrm{NaHCO}_{3}$ neutralizes all carboxylic groups, $\mathrm{Na}_{2} \mathrm{CO}_{3}$-carboxylic and lactonic groups- and $\mathrm{NaOH}$-carboxylic, lactonic and phenolic groups-. The total number of basic sites was determined with $0.05 \mathrm{~N} \mathrm{HCl}$ [15]. The procedure is the same as above mentioned, as back-titration of the excess of $\mathrm{HCl}$ was performed using a $0.05 \mathrm{~N} \mathrm{NaOH}$ solution.

\subsection{Spin Trapping Electron Spin Resonance (ESR) Measurements}


The formation of paramagnetic species during irradiation of carbon suspensions in aqueous solution was detected by Spin Trapping ESR measurements, using 5,5-dimethylpyrroline-Noxide (DMPO) as trapping agent [16]. About $2.5 \mathrm{mg}$ of the samples were suspended in $5 \mathrm{ml}$ of $\mathrm{HClO}_{4}$ buffer at $\mathrm{pH} 3$ (final solids concentration $0.5 \mathrm{~g} / \mathrm{L}$ ), and $10 \mu \mathrm{L}$ of DMPO was added to the suspension to reach a final concentration of $18 \mathrm{mM}$, introduced in quartz tubes and irradiated for 20 minutes (Philips TL K40W/05 lamp, with a broad emission peak centred at $365 \mathrm{~nm}$ ). ESR spectra were immediately recorded from the solution (after filtering out the solids) at room temperature on a Bruker ESP 300E X band spectrometer with the following spectral parameters: receiver gain 105; modulation amplitude $0.52 \mathrm{G}$; modulation frequency $100 \mathrm{KHz}$, microwave frequency $9.69 \mathrm{GHz}$; microwave power $5.024 \mathrm{~mW}$; conversion time $40.96 \mathrm{~ms}$; center field $3450 \mathrm{G}$, sweep width $120 \mathrm{G}$. Simulations of the individual components of the ESR spectra were obtained using the Winsim 2002 software [17].

\section{Results and discussion}

The choice of the pristine carbon used in this work was based on its physico-chemical features (Table 1) and its good performance towards phenol photooxidation in the absence of semiconductors after 6 hours of irradiation [9]. Furthermore, this adsorbent is commonly used for the removal of phenolic compounds in the purification of industrial wastewater and drinking water. Although phenol photodegradation efficiency in solution has been previously reported for carbon $\mathrm{CV}$, herein we investigate the performance under long illumination times during consecutive photodegradation cycles. Also the chemical composition (surface chemistry and low ash content, as seen in Table 1 and Fig. S2) makes it a good candidate to explore the role of the surface chemistry on phenol photooxidation reaction upon cycling. The outcome of previous studies is used here only for data interpretation.

Due to the characteristics of our irradiation source, the photolytic breakdown of phenol under our conditions was rather large (see Fig. S3); however the overall efficiency and mineralization extent of direct photolysis is very poor (TOC value drop by $8 \%$ after $3 \mathrm{~h}$ ) because apart phenol, none of the intermediates are further decomposed. As the yield of direct photolysis is highly dependent on the concentration from solution [18], the photocatalytic cycles were designed so as to maintain a constant phenol solution at the initial stage of the illumination step (thereby controlling the effect of the photolysis for all the studied materials). Also, based on their porous nature and different surface chemistry (Table 1), these carbons are expected to show different adsorption capacities. This is expected to modify the concentration 
of phenol in solution in the course of the photooxidation reaction and should be taken into account to compare their performance on consecutive photodegradation cycles (discriminating between the photooxidation reaction and the adsorption, both occurring simultaneously), as well as to compare vs non porous titania powders as reference catalysts $\left(\mathrm{S}_{\mathrm{BET}}=50 \mathrm{~m}^{2} / \mathrm{g}\right)$, in which the amount of phenol adsorbed is almost negligible $(\sim 3 \%)$.

Indeed, Fig. 2 shows the kinetics of adsorption and equilibrium adsorption isotherms of phenol obtained at dark conditions on both carbons, where their different adsorption behavior is evidenced. A summary of the Langmuir adsorption parameters and surface coverage is compiled in Table S1 and Fig. S4. The adsorption kinetics was very fast, with the maximum uptake attained after 20-30 minutes in both carbons. This is consistent with their well developed micro/mesoporous network as shown in Table 1; even after thermal treatment the porosity remained rather unchanged, beyond a slight drop or the total pore volume but preserving the micropore to mesopore ratio. This slight structural annealing upon heating at high temperatures is rather common on carbon materials [19, 20]. On the other hand, the amount adsorbed is almost twice larger on carbon $\mathrm{CVH}$ compared to $\mathrm{CV}$. This is also consistent with its more basic nature, as phenol is favorably adsorbed in hydrophobic carbons [21-24].
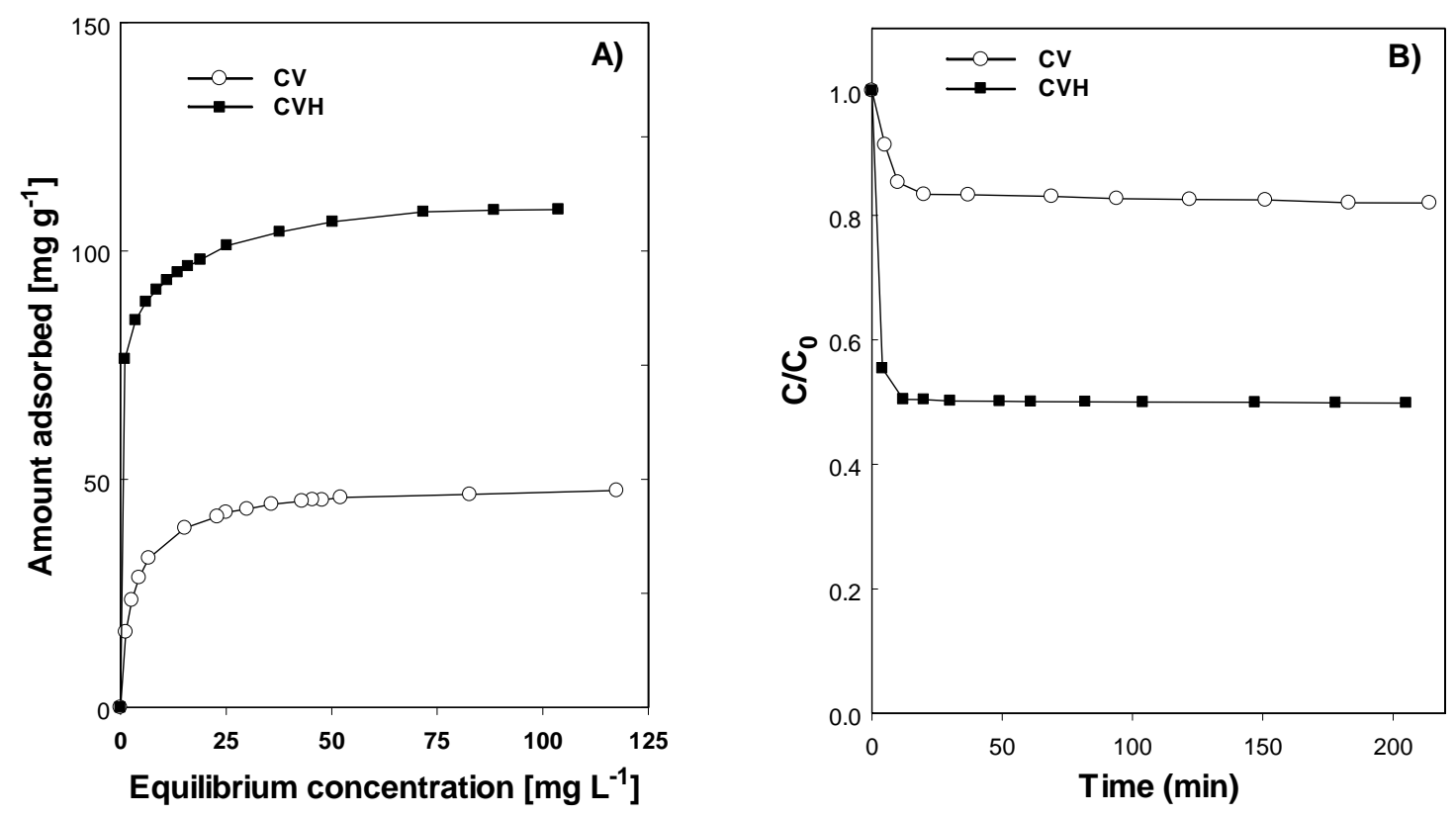

Fig. 2. Equilibrium adsorption isotherms of phenol (A) and kinetics of adsorption (B) under dark conditions on carbons $\mathrm{CV}$ and $\mathrm{CVH}$. 
For all these reasons, and to avoid biased interpretations, the photocatalytic experiments were designed allowing the carbons to equilibrate in contact with the phenol solution for $30 \mathrm{~min}$ before exposing to UV light. The initial phenol concentration was calculated for each carbon considering the equilibrium adsorption isotherms (Fig. 2), so as to obtain a concentration of $50 \mathrm{ppm}$ in solution after the equilibration step. As mentioned above, this will keep the direct photolysis constant in all the experiments. Furthermore, it is important to point out that, even though both carbons have different adsorption capacities, for both materials the chosen experimental conditions correspond to an almost full coverage of the carbon surface, with respect to their corresponding maximum uptake. For instance, the amount adsorbed accounting for $50 \mathrm{ppm}$ phenol equilibrium concentration is ca. 46 and $116 \mathrm{mg} / \mathrm{g}$ for $\mathrm{CV}$ and CVH, respectively, which correspond to a surface coverage of 93 and $96 \%$ for both carbons (Fig. S4). This is most important since in previous works we have demonstrated that the photocatalytic degradation of the pollutant adsorbed inside the porosity of the carbons also occurs upon irradiation in solution [9]. Thus, under these experimental conditions the direct photolysis and the degradation rate of the confined species on the porosity maintained constant for both carbons. This should be advantageous for a direct comparison of the overall photocatalytic yield upon cycling of the activated carbons monitored in solution.

\subsection{First photocatalytic cycle}

Fig. 3 and 4 show the evolution of phenol concentration and its degradation intermediates after several hours of illumination for the carbon materials compared to titania powders. The first cycle, discussed in this subsection, is compared for the three materials for clarity purposes. Experiments were initially conducted in the presence of air but without an external supply of oxygen (ambient conditions). When comparing the performance of the carbon materials for phenol photo-oxidation to that of titanium dioxide, several differences become clear. First, the overall phenol conversion after one cycle (i.e. $180 \mathrm{~min}$ ) is rather high for all the processes, with slight higher values for both activated carbons compared to titania. Since the photocatalytic runs were preceded by an equilibration step and thus the porous voids of the activated carbons are already filled with phenol molecules when the irradiation starts, it seems reasonable to consider that the disappearance of the pollutant from the solution under these conditions is mainly due to a photocatalytic process. 
Secondly, the total amount of degradation intermediates was again slightly lower for both carbon materials and the distribution of degradation intermediates was also dependent on the type of catalyst, with a marked regioselective photoxidation for the activated carbons and the titania powders. For instance, oxidation of phenol in para- position to form quinones (hydroquinone and benzoquinone) is predominant when using titania as catalyst (under these operating conditions), as opposed to preferential ortho- position (1,2 catechol) in the case of both activated carbons. The regioselective formation of catechol over quinones is considered more advantageous for the overall reaction yield; according to literature, catechols are more reactive than quinones (due to the stability of intermediates formed) $[25,26]$, as a result of which the degradation pathway of catechol proceeds through a simple mechanism involving a less number of intermediates (organic acids).
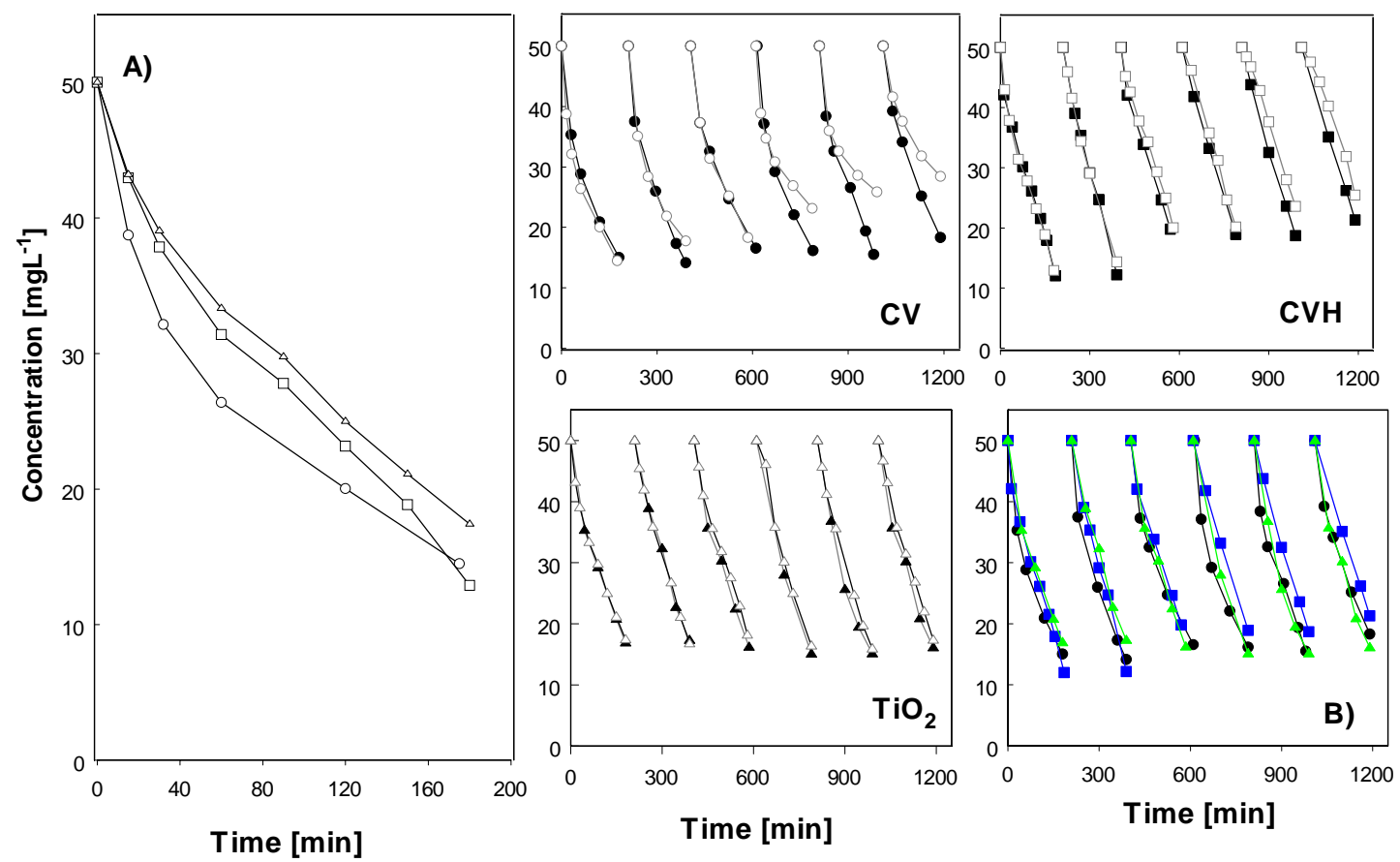

Fig. 3. Evolution of phenol concentration after several consecutive photocatalytic cycles on carbon $\mathrm{CV}$, carbon $\mathrm{CVH}$ and $\mathrm{TiO}_{2}$ under excess (solid symbols) and depleted (empty symbols) oxygen supply. For clarity, comparison of the performance of the three studied materials is shown for (A) the first and (B) all the cycles; circles (CV), squares $(\mathrm{CVH})$, triangles $\left(\mathrm{TiO}_{2}\right)$.

In all three cases negligible amounts of the meta-hydroxylated compound (resorcinol) were detected. Interestingly, trace amounts of a trihydroxylated intermediate $(1,2,4$ 
trihidroxybenzene) were formed in the catalyzed reactions (but not upon direct photolysis). The formation of this trihydroxylated derivative seems to be somehow related to the acidity/basicity of the catalysts, as larger amounts were detected for both $\mathrm{CV}$ and $\mathrm{TiO}_{2}$ (hydrophilic materials), and almost negligible amounts for the more hydrophobic carbon $(\mathrm{CVH})$. No traces of other phenol coupling subproducts were detected despite they are rather frequent upon direct hydroxylation by free radical reagents in solution [25].

The different distribution of hydroxylated degradation intermediates in the presence of carbon materials in the first cycle is in good agreement with the data reported in the literature $[5,9$, $13,27,28]$. In most cases the participation of catechol and quinones is described, and scarce mention is found to trihydroxylated products, although they have been detected as primary and secondary hydroxylation intermediates at quite small concentrations [29, 30].
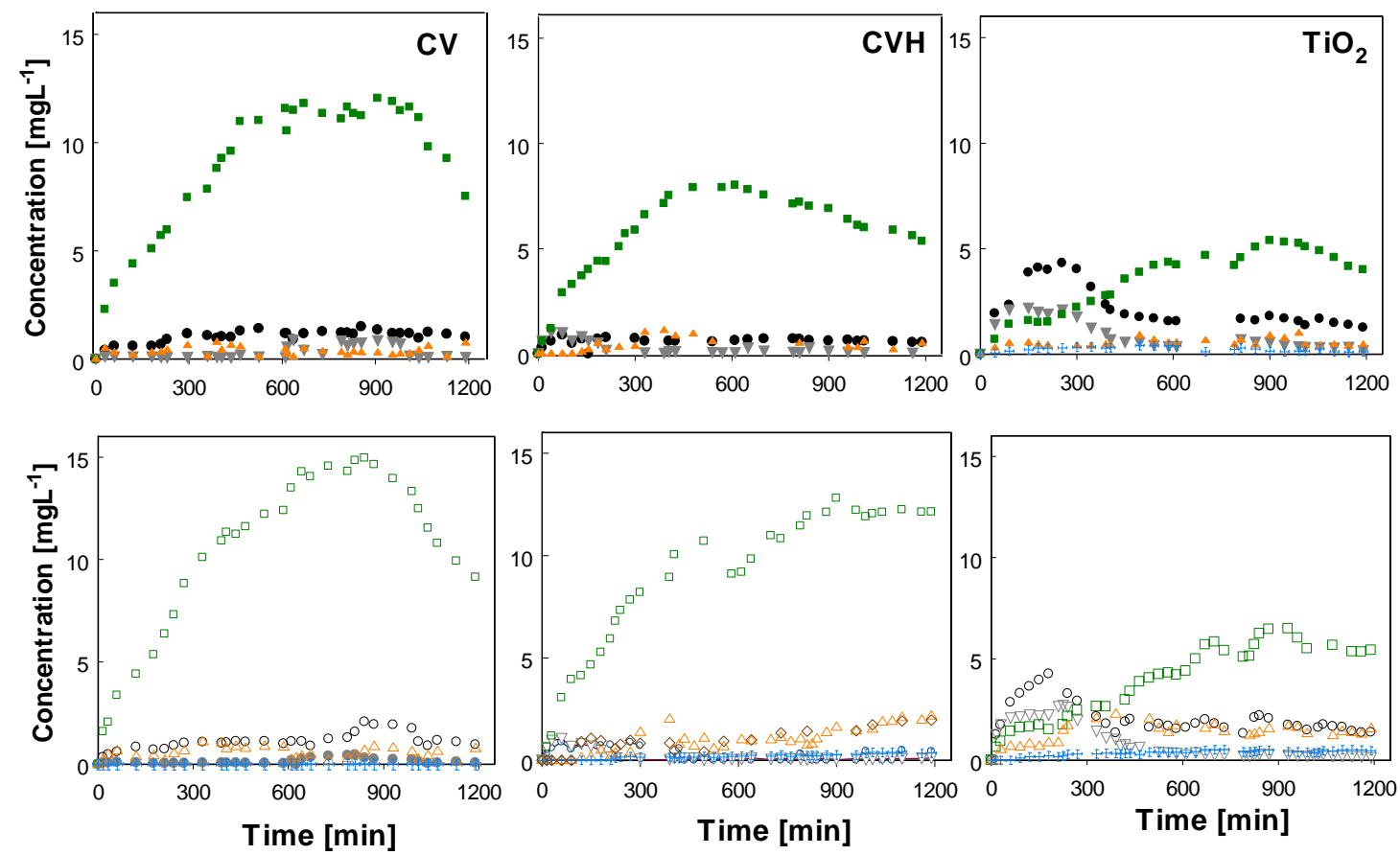

Fig. 4. Evolution of main phenol photooxidation intermediates detected in solution upon the consecutive photocatalytic cycles on the studied materials under excess (top, solid symbols) and depleted (bottom, empty symbols) oxygen supply. Hydroquinone (circles); benzoquinone (down triangles); catechol (squares); 2,4,6trihydroxybenzene (up triangles); resorcinol (crosses); 1,3,5-trihydroxybenzene (diamonds). 
Considering that the ortho- and para- substitution are the preferred positions in electrophilic substitution of aromatic rings, it seems reasonable that the formation of the hydroxylated products follows an electrophilic mediated pathway or an electron transfer process promoted by hydroxyl radicals, direct hole-oxidation, or some other transient one-electron oxidants [31, 32]. The poor oxidation extent of the intermediates -compared to phenol- indicates the preferential reactivity of the radicals towards the aromatic ring, rather than to phenol subproducts [31], which is also characteristic of electrophilic mechanisms. A similar photodegradation pathway via electrophilic substitution of the aromatic ring has been reported for 4-chlorophenol [33].

It should also be mentioned that electrophilic additions are favoured at acidic $\mathrm{pH}$ [31], which is consistent with the higher oxidation yields obtained for $\mathrm{TiO}_{2}$ and $\mathrm{CV}$, both acidic materials $(\mathrm{COOH}$ moieties in the carbon are Bronsted acid sites, whereas $\mathrm{Ti}$ atoms with oxygen vacancies are Lewis acid sites). In the case of $\mathrm{CVH}$, its more basic nature could delay the electrophilic substitution reaction, due to the increased basicity of the reaction medium in contact with the more hydrophobic carbon which adsorbs $\mathrm{H}^{+}$.

The analysis of the TOC values from solution after 3 hours of irradiation (Table 2) shows a similar trend, with rather smaller values for carbon $\mathrm{CVH}$. Indeed, the mineralization degree after the first cycle (accounting for the mass balance evaluated from the initial and final TOC values of the corresponding cycle) was about $50 \%$ for $\mathrm{TiO}_{2}$ and $\mathrm{CVH}$, slightly higher than the $42 \%$ for sample CV. This points out the better performance of the carbons (at least) in the first cycle, not only in terms of phenol conversion but also in the complete mineralization.

Table 2. Total Organic Carbon (TOC, $\mathrm{mg} \mathrm{C} / \mathrm{L}$ ) values in solution after the consecutive photocatalytic cycles. Shaded values correspond to the experimental carried out under excess of $\mathrm{O}_{2}$. Initial TOC value was $38 \mathrm{mg} \mathrm{C} / \mathrm{L}$ for all the samples.

\begin{tabular}{lllllll}
\hline & \multicolumn{2}{c}{ Cycle 1 } & \multicolumn{3}{c}{ Cycle 3 } & \multicolumn{2}{c}{ Cycle 6 } \\
\hline CV & 22.2 & 22.2 & 47.5 & 40.2 & 66.4 & 36.4 \\
CVH & 18.1 & 18.1 & 43.6 & 38.3 & 51.2 & 40.0 \\
TiO $_{2}$ & 19.3 & 19.3 & 30.5 & 29.6 & 49.8 & 45.3 \\
\hline
\end{tabular}

\subsection{Consecutive photocatalytic cycles}

On the other hand, the situation changes when the materials are submitted to consecutive illumination cycles (Fig. 3 and 4). The performance of the activated carbons gradually 
decreases with the number of the cycles, being this effect more remarkable in the acidic carbon (sample CV). After the third run the overall phenol efficiency reaches a plateau at about $50 \%$ conversion after the first $90 \mathrm{~min}$ of irradiation in every run. This is in agreement with the accumulation of intermediates observed for the first cycles, where it seems that the materials are capable of promoting the photodegradation of phenol but not that of the polyhydroxylated intermediates.

For titania powder the tendency seems to be more stable (Fig. 3 and 4); although the conversion of phenol still reaches values around $60 \%$ upon cycling, the degradation of the intermediates seems to be less efficient (or delayed) with the number of cycles, as an important accumulation of hydroxylated intermediates was also detected after $400 \mathrm{~min}$ (2-3 cycles). The preferential oxidation to quinones dominates the photocatalytic reaction of $\mathrm{TiO}_{2}$ showing a maximum concentration in the first 4 hours, followed by a gradual fall below 1-3 ppm. After this point, the amount of catechol started to accumulate in solution with the number of cycles, following the same pattern described for both carbons.

Although some differences are evident in the evolution of the TOC values within the cycles (Table 2), the three samples perform quite similarly in terms of degree of mineralization after the sixth cycle. The total organic carbon loading treated in the whole process (after the 6 cycles) was about $228 \mathrm{mg} \mathrm{C} / \mathrm{L}$, leading to very close mineralization yields for samples $\mathrm{TiO}_{2}$, CVH and CV: 78, 78 and 71\%, respectively. This leads us to conclude that the photocatalytic efficiency of the carbon is comparable to that showed by the commercial titania. This contrasts with the different trend of phenol disappearance from solution showed in Fig. 3, and the higher accumulation of aromatic intermediates when irradiating the activated carbon (Fig. 4). Both results indicate that the accumulation of short alky chain acids (final degradation intermediates during phenol photo-oxidation) would be higher for titanium oxide than for the activated carbons.

We attributed the fall in the efficiency to an eventual depletion of the dissolved oxygen present in the solution during the cycles, since they were carried out in the presence of air but without an external supply of oxygen. The influence of molecular oxygen will be further discussed below.

Furthermore, molecular oxygen is known to be a good electron scavenger to generate superoxide anions, which could contribute to the oxidation of the pollutant in solution. The formation of O-radicals upon illumination of suspensions of the activated carbons was confirmed by Electron Spin Resonance spectroscopy, using DMPO as spin trap (Fig. 5). The ESR spectra of all three samples showed the characteristic quartet peak profile with 1:2:2:1 
intensity $(\mathrm{g}=2.006, \mathrm{aN}=\mathrm{a} \beta \mathrm{H}=14.8 \mathrm{G}$ hyperfine splitting constants $)$, due to the formation of DMPO-OH adducts $[16,34]$ attributed to the presence of hydroxyl and/or superoxide radicals in the reaction medium (superoxide anion is unstable in water and is rapidly converted to $\cdot \mathrm{OH}$ radical, eventually contributing to the DMPO-OH adduct signal) [34,35]. Simulation of the experimental spectra allowed the identification of a second adduct for titanium dioxide, (HDMPO-OH adduct, $\mathrm{aN}=14.6 \mathrm{G}, \mathrm{a} \gamma \mathrm{H}=1.1 \mathrm{G}$ ) [34], with a relative abundance close to $20 \%$. These adducts were not detected when the DMPO solution was illuminated alone, confirming that they are generated during irradiation of herein studied catalysts and corroborating the high reactivity in the presence of molecular oxygen.
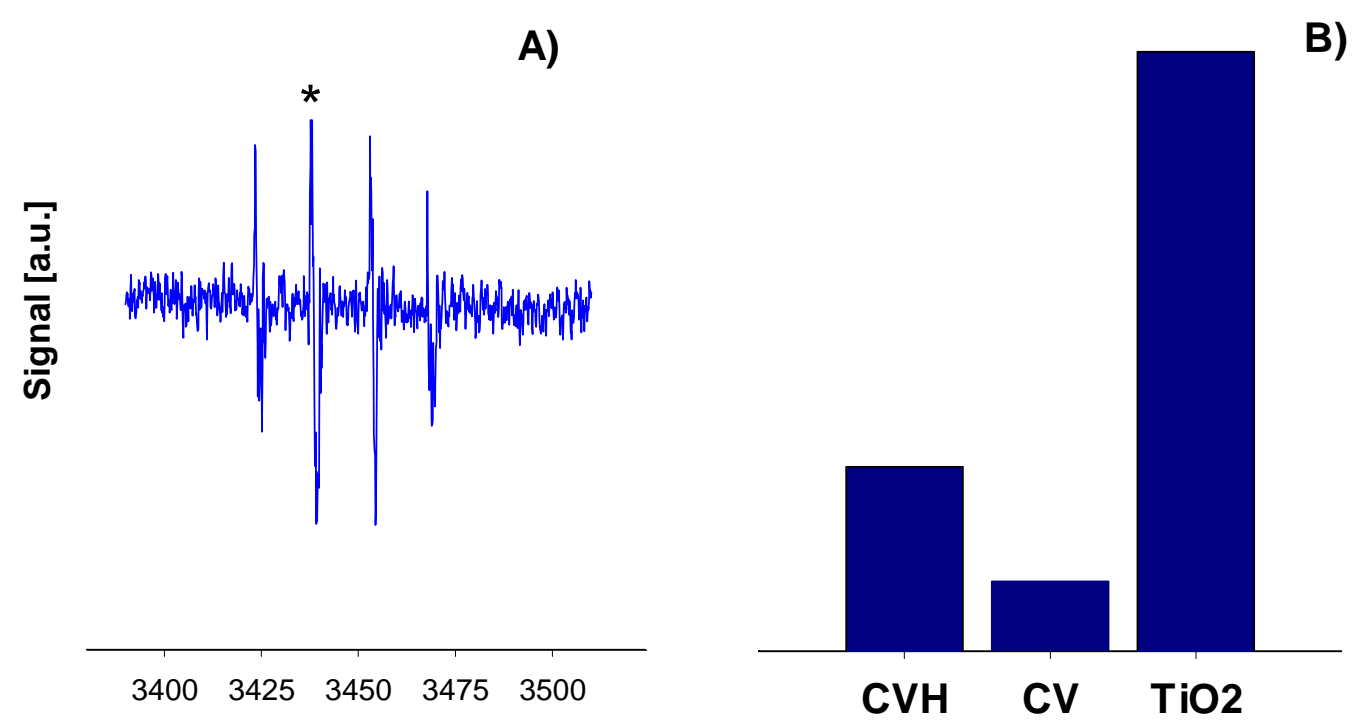

Magnetic Field [Gauss]

Fig. 5. (A) Example of the ESR signal measured after UV irradiation of aqueous suspensions of the activated carbons and titania powders. (B) Quantification of the radical species corresponding to DMPO-OH adducts by integration of the intensity of the second peak in the profiles, as marked by the star.

Moreover, integration of the intensity of the second peak in the profiles allowed the quantification of the radical species (Fig. 5B). Although lower concentrations were obtained in the carbons, compared to $\mathrm{TiO}_{2}$, it is interesting to recall that the amount of radicals was larger for $\mathrm{CVH}$ than $\mathrm{CV}$. This suggests that the presence of O-containing functionalities on the carbon matrix has a negative impact on the detection of radicals. A similar behavior with the extent of the carbon oxidation has been reported for other carbons [11]. It should be noted that despite the low concentration of radicals detected in solution, carbon $\mathrm{CV}$ displayed 
similar activity towards phenol photooxidation than CVH (Fig. 3 and 4), being higher than other carbons with larger ESR signals [11, 12].

\subsection{Consecutive photocatalytic cycles under excess of oxygen}

The role of the oxygen as a promoter of phenol photooxidation was further confirmed measuring the cycles under continuous oxygen supply (Fig. 3 and 4), to avoid an eventual depletion of dissolved oxygen in solution during the cycling.

The loss of activity towards phenol conversion after consecutive cycles was less pronounced under excess of oxygen, particularly for carbon $\mathrm{CV}$ that had shown a marked deactivation upon cycling. In fact, the performance of both carbons was similar to that of titanium dioxide. As for the intermediates, the amount detected is lower when the cycles are carried out under excess of oxygen (Fig. 4), confirming that dissolved oxygen is consumed during the photocatalytic reaction, likely through the formation of O-radicals. Also, the regioselectivity towards the formation of catechol over quinones in the activated carbons is still maintained. The enhanced photocatalytic conversion under excess of oxygen is also in agreement with TOC values (Table 2) that show a higher mineralization of phenol.

Based on these results, it can be assumed that the formation of O-radicals upon illumination of the catalysts is important to achieve high phenol conversions and mineralization. Direct hole oxidation of phenol to form catechol and trihydroxylated intermediates should not be discarded [37], although this reaction typically displays a slow kinetics [38]. With increasing the concentration of dissolved oxygen, the rate for the recombination of the photogenerated species is reduced due to the competitive electron scavenging by oxygen; as a result, the generation of O-radicals would be improved. The effect was very marked in the hydrophilic carbon, but it was evident also for $\mathrm{CVH}$ and the titania powders (Fig. 5). A similar role of dissolved oxygen has been reported for the mineralization of phenol in aqueous $\mathrm{TiO}_{2}$ suspensions [39,40]. Moreover, TOC values (Table 2) revealed that the degree of mineralization also depended on the supply of oxygen introduced to the photoreactor, being the differences higher after longer irradiation times (i.e., increasing the number of cycles).

Although some of the intermediates detected have more toxicity than phenol itself [41], they are detected at a trace level after six cycles (Fig. 4); in all cases at much lower values compared to the overall phenol concentration treated during the course of the reaction (ca. 300 ppm).

The porosity of the carbons decreased gradually with the number of cycles, as shown in Fig. 6, indicating that further adsorption occurs to some extent in the course of the 
photooxidation experiments. The clogging of the porosity affected mostly the specific surface area and microporosity, which is somehow expected since both textural parameters are closely related and micropores are known to be the active sites where phenol and its aromatic photo-oxidation products are preferably adsorbed [21]. The collapse of the porosity upon cycling was more pronounced for sample $\mathrm{CV}$, indicating that a higher amount of species are adsorbed inside its porous network. This result was somehow expected taking into account that this carbon leads to lower photodegradation and mineralization yields than sample CVH. It has to be considered that a greater amount of remaining phenol and its oxidation products are present in solution when irradiating carbon $\mathrm{CV}$, favoring the extension of the adsorption.

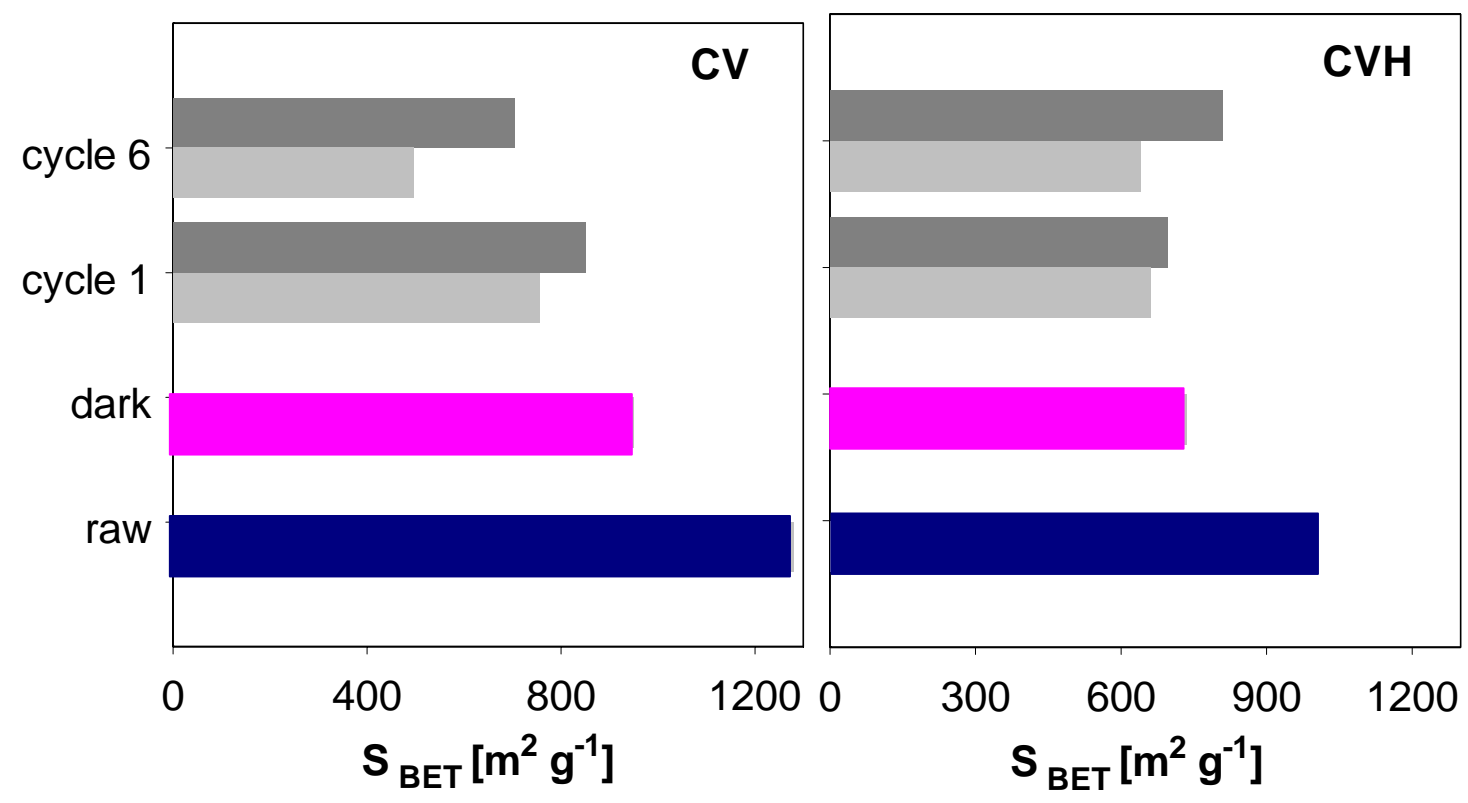

Fig. 6. Evolution of the porous features of the catalysts upon consecutive irradiation cycles under depleted (light grey) and excess (dark grey) dissolved oxygen conditions for the initial carbons, after adsorption at dark conditions and after consecutive photocatalytic runs (first and sixth cycles).

Moreover, the evolution of the porous features of the carbons during the consecutive cycles was very dependent on the presence of dissolved oxygen in the reactor. Under excess of oxygen, the collapse of the porosity upon consecutive runs is significantly lower, which confirms that in these conditions phenol and its degradation intermediates are photo oxidized rather than adsorbed in the porosity of the carbons (which would lead to lower porous features). These results are in agreement with the higher photodegradation performance 
(Table 2 and Fig. 3 and 4), and thus corroborate the outstanding role of dissolved oxygen as a promoter of phenol photooxidation.

\section{CONCLUSIONS}

The performance of activated carbons with different surface chemistry after 20 hours of illumination in consecutive photocatalytic cycles for the photodegradation of phenol has been evaluated and compared to that of commercial $\mathrm{TiO}_{2}$ under similar experimental conditions. The overall performance of the activated carbons upon cycling was comparable to that of commercial titania -especially in conditions of excess of oxygen- with close mineralization yields in all three studies materials after six consecutive cycles (ca. 20 hours of illumination). Data showed a strong dependence of the photocatalytic efficiency on the basic/acidic nature of the activated carbons, with a lower performance for the hydrophilic carbon. For the activated carbons, a marked accumulation of phenol degradation intermediates was observed during cycling, with preferential formation of catechol over quinones. For titanium oxide, the concentration of aromatic intermediates is lower but still TOC values show low mineralization due to the accumulation of short alkyl chain organic acids.

Phenol conversion and mineralization rates were greatly enhanced in the presence of excess of dissolved oxygen in the solution, demonstrating the outstanding role of oxygen in the photooxidation of phenol. This was critical for the long-term performance of the hydrophilic carbon, which showed a sharp fall in phenol conversion upon cycling under oxygen depletion conditions. The evolution of the porous features of the carbons was also very dependent on the presence of dissolved oxygen. The lower collapse of the porosity under oxygen excess indicates a higher conversion of phenol and its degradation intermediates. Furthermore, the formation of O-radicals upon illumination of both activated carbons and titanium dioxide was evidenced by electron spin resonance spectroscopy. Both results confirmed that the formation of $\bullet \mathrm{OH}$ and $\cdot \mathrm{O}_{2}{ }^{-}$is important to achieve high phenol conversions and mineralization yields, particularly for activated carbons.

\section{ACKNOWLEDGMENTS}

The authors thank the financial support of the Spanish MINECO (grants CTM2008/01956, CTM2011/02338) and PCTI Asturias (Fondos Feder 2007-2013, grant PC10-002). LFV and RJC thank CSIC and PCTI Asturias for their JAE-Pre and Severo-Ochoa fellowships, respectively. The authors are grateful to Dr. Maurino and Dr. Laurenti from the University of 
Torino for the ESR measurements and to Ms. Querejeta-Montes for her help in the adsorption kinetics.

\section{REFERENCES}

[1] Serpone N, Pelizzetti E (Eds) in Photocatalysis: fundamental and applications, ed. Pelizzetti E and Serpone N, New York: Wiley Interscience; 1989.

[2] Malato S, Fernández-Ibáñez P, Maldonado MI, Blanco J, Gernjak W. Decontamination and disinfection of water by solar photocatalysis: recent overview and trends. Catal. Today 2009; 147:1-59.

[3] Leary R, Westwood A. Carbonaceous nanomaterials for the enhancement of $\mathrm{TiO}_{2}$ photocatalysis. Carbon 2011;49:741-72.

[4] Faria JL, Wang W. Carbon materials in photocatalysis. In: Serp P, Figueiredo JL, editors. Carbon materials for catalysis. New York; John Wiley \& Sons; 2009 p. 481506.

[5] Matos J, Laine J, Herrmann JM. Synergy effect in the photocatalytic degradation of phenol on a suspended mixture of titania and activated carbon. Appl. Catal. B: Environ 1998;18:281-91.

[6] Matos J, Garcia A, Cordero T, Hovelon JM, Ferronato C, Eco-friendly $\mathrm{TiO}_{2}-\mathrm{AC}$ Photocatalyst for the Selective Photooxidation of 4-Chlorophenol, Catal Lett 2009;130:568-74.

[7] Araña J, Doña-Rodríguez JM, Tello Rendón E, Garriga i Cabo C, González-Díaz O, Herrera-Melián JA, et al. $\mathrm{TiO}_{2}$ activation by using activated carbon as a support. Part I. Surface characterisation and decantability study Appl Catal B: Environ 2003; 44:16172.

[8] Henderson MA. A surface science perspective on $\mathrm{TiO}_{2}$ photocatalysis, Surf. Sci. Rep. 2011;66:185-297.

[9] Velasco LF, Fonseca IM, Parra JB, Lima JC, Ania CO. Photochemical behaviour of activated carbons under UV irradiation. Carbon 2012;50:249-58.

[10] Ania CO, Velasco LF, Valdes-Solis T. Photochemical response of carbon materials. In Novel Carbon Adsorbents. Tascon JMD, editor. London; Elsevier; 2012 p. 521-47.

[11] Velasco LF, Maurino V, Laurenti E, Ania CO. Light-induced generation of radicals on semiconductor-free carbon photocatalysts. Appl Catal A: Gen 2013;453:310-5. 
[12] Velasco LF, Maurino V, Laurenti E, Fonseca IM, Lima JC, Ania CO. Photoinduced reactions occurring on activated carbons. A combined photooxidation and ESR study Appl Catal A: Gen 2013;452:1-8

[13] Matos J, Laine J, Herrmann JM. Uzcategui D, Brito JL, Influence of activated carbon upon titania on aqueous photocatalytic consecutive runs of phenol photodegradation, Applied Catalysis B: Environmental 2007;70:461-9.

[14] Boehm HP, in: DD Eley, H Pines, PB Weisz (Eds.), Chemical Identification of Surface Groups, Advances in Catalysis, vol. 16, Academic Press, New York, 1966, p. 179-274.

[15] Papirer E, Li S, Donnet JB. Contribution to the study of basic surface groups on carbons, Carbon 1987;25:243-7.

[16] Finkelstein E, Rosen GM, Rauckman E. Spin trapping. Kinetics of the reaction of superoxide and hydroxyl radicals with nitrones. J. Arch. Biochem. Biophys.1980;200:116.

[17] Duling DR. Simulation of Multiple Isotropic Spin Trap EPR SpectraJ. Magn. Res. Series B 1994;104:105-10.

[18] Shanmuga Priya S, Premalatha M, Subramanian P. Elucidation of Kinetics of Photolysis of Phenol, IE(J) Chem. Eng. 2009;90:10-6.

[19] Rao AM, Fung AWP, Dresselhaus DS, Endo M. Structural characterization of heattreated activated carbon fibers, J. Mater. Res. 1996;7:1788-94.

[20] Ania CO, Menendez JA, Parra JB, Pis JJ, Effect of microwave and conventional regeneration on the microporous and mesoporous network and on the adsorptive capacity of activated carbons, Microp. Mesop. Mater. 2005;85:7-15.

[21] Velasco LF, Ania CO, Understanding phenol adsorption mechanisms on activated carbons, Adsorpt. 2010;17:247-54.

[22] Moreno-Castilla C. Adsorption of organic molecules from aqueous solutions on carbon materials, Carbon 2004;42:83-94.

[23] Terzyk J. Further insights into the role of carbon surface functionalities in the mechanism of phenol adsorption, J. Colloid Interf Sci 2003;268:301-29.

[24] Nevskaia DM, Guerrero-Ruiz A. Comparative study of the adsorption from aqueous solutions and the desorption of phenol and nonylphenol substrates on activated carbons, J Colloid Interf. Sci. 2001;234:316-21.

[25] Ross L, Barclay C, Vinqvist MR. Phenols as antioxidants. In Z. Rappoport, editor. The Chemistry of Phenols. England; John Wiley \& Sons; 2003 p. 839-909. 
[26] Foti M, Ruberto G. Kinetic solvent effects on phenolic antioxidants determined by spectrophotometric methods. J. Agric. Food Chem. 2001;49:342-8.

[27] Santos A, Yustos P, Quintanilla A, Rodríguez S, Garcia-Ochoa F. Route of the catalytic oxidation of phenol in aqueous phase. Appl Catal B: Environ 2002;39:97-113.

[28] Velasco LF, Parra JB, Ania CO, Role of activated carbon features on photodegradation of phenol, Appl. Surf. Sci. 2010;256:5254-8.

[29] Salaicesa M, Serrano B, Lasa HI. Photocatalytic conversion of phenolic compounds in slurry reactors, Chem. Eng. Sci. 2004;59:3-15.

[30] Al-Ekabi H, Serpone N. Kinetic studies in heterogeneous photocatalysis. 1. Photocatalytic degradation of chlorinated phenols in aerated aqueous solutions over $\mathrm{TiO}_{2}$ supported on a glass matrix. Journal of Physical Chemistry 1988;92:5726-31.

[31] Prakash Reddy V, Surya Prakash GK. Electrophilic reactions of phenols. In Z. Rappoport, editor. The Chemistry of Phenols. England; John Wiley \& Sons; 2003 p. $600-60$.

[32] Kutty TRN, Ahuja S. Retarding effect of surface hydroxylation on titanium(IV) oxide photocatalyst in the degradation of phenol. Mat. Res. Bull. 1995;30:233-41.

[33] Matos J, Garcia A, Poon PS. Environmental green chemistry applications of nanoporous carbons. J. Mater. Sci. 2010;45:4934-44.

[34] Makino K, Hagi A, Ide H, Murakami A, Nishi M. Can. J Chem. 1992;70:2818-27.

[35] Finkelstein E, Rose GM, Rauckman EJ, Paxton J. Spin trapping of superoxide, J. Mol Pharmacol. 1979;16:676-85.

[36] Gray B, Carmichael AJ, Kinetics of superoxide scavenging by dismutase enzymes and manganese mimics determined by electron spin resonance, Biochem. J. 1992;281:795802.

[37] Hoorspoll WM, Photochemistry of Phenols. In Z. Rappoport, editor. The Chemistry of Phenols. England; John Wiley \& Sons; 2003 p. 839-909.

[38] Schwarz PF, Turro NJ, Bossmann SH, Braun AM, Wahab A, Durr H, A new method to determine the generation of hydroxyl radicals in illuminated $\mathrm{TiO}_{2}$ suspensions, J. Phys. Chem. B 1997;101:7127-34.

[39] Okamoto K, Yamamoto Y, Tanaka M, Itaya A. photocatalytic decomposition of phenol over $\mathrm{TiO}_{2}$ powder, Bull. Chem. Soc. Jpn. 1985;58:2015-22.

[40] Minero C, Bedini A, Maurino V, Glycerol as a probe molecule to uncover oxidation mechanism in photocatalysis, Appl. Catal. B: Environ. 2012;128:135-43. 
[41] Windholz M (Ed) in The Merck Index: Encyclopedia of Chemicals, Drugs and Biologicals, New Jersey: Merck \& Co., Inc. 1983.

\section{Figures Captions}

Fig. 1. Sketch illustrating the experimental procedure followed for the consecutive phenol photooxidation cycles on sample CVH. Arrows indicate the beginning/end of the irradiation period.

Fig. 2. Equilibrium adsorption isotherms of phenol (A) and kinetics of adsorption (B) under dark conditions on carbons $\mathrm{CV}$ and $\mathrm{CVH}$.

Fig. 3. Evolution of phenol concentration after several consecutive photocatalytic cycles on carbon $\mathrm{CV}$, carbon $\mathrm{CVH}$ and $\mathrm{TiO}_{2}$ under excess (solid symbols) and depleted (empty symbols) oxygen supply. For clarity, comparison of the performance of the three studied materials is shown for (A) the first and (B) all the cycles; circles (CV), squares $(\mathrm{CVH})$, triangles $\left(\mathrm{TiO}_{2}\right)$.

Fig. 4. Evolution of main phenol photooxidation intermediates detected in solution upon the consecutive photocatalytic cycles on the studied materials under excess (top, solid symbols) and depleted (bottom, empty symbols) oxygen supply. Hydroquinone (circles); benzoquinone (down triangles); catechol (squares); 2,4,6trihydroxybenzene (up triangles); resorcinol (crosses); 1,3,5-trihydroxybenzene (diamonds).

Fig. 5. (A) Example of the ESR signal measured after UV irradiation of aqueous suspensions of the activated carbons and titania powders. (B) Quantification of the radical species corresponding to DMPO-OH adducts by integration of the intensity of the second peak in the profiles, as marked by the star.

Fig. 6. Evolution of the porous features of the catalysts upon consecutive irradiation cycles under depleted (light bars) and excess (dark bars) dissolved oxygen conditions for the initial carbons, after adsorption at dark conditions and after consecutive photocatalytic runs (first and sixth cycles).

\section{Tables Captions}

Table 1. Main physicochemical characteristics of the selected activated carbons obtained from gas adsorption, elemental analysis, point of zero charge, Boehm titration and TPD-MS. 
Table 2. Total Organic Carbon (TOC, mg C/L) values in solution after the consecutive photocatalytic cycles. Shaded values correspond to the experimental carried out under excess of $\mathrm{O}_{2}$. Initial TOC value was $38 \mathrm{mg} \mathrm{C} / \mathrm{L}$ for all the samples. 